\title{
Role-Based Secure Inter-operation and Resource Usage Management in Mobile Grid Systems
}

\author{
Antonios Gouglidis and Ioannis Mavridis \\ Department of Applied Informatics, University of Macedonia, \\ 156 Egnatia Str., 54006, Thessaloniki, Greece \\ \{agougl, mavridis\}@uom.gr
}

\begin{abstract}
Dynamic inter-domain collaborations and resource sharing comprise two key characteristics of mobile Grid systems. However, interdomain collaborations have proven to be vulnerable to conflicts that can lead to privilege escalation. These conflicts are detectable in interoperation policies, and occur due to cross-domain role relationships. In addition, resource sharing requires to be enhanced with resource usage management in virtual organizations where mobile nodes act as resource providers. In this case the enforcement of resource usage policies and quality of service policies are required to be supported due to the limited capabilities of the devices. Yet, the ANSI INCITS 359-2004 standard RBAC model provides neither any policy conflict resolution mechanism among domains, nor any resource usage management functionality. In this paper, we propose the domRBAC model for access control in mobile Grid systems at a low administrative overhead. The domRBAC is defined as an extension of the standardized RBAC by incorporating additional functionality to cope with requirements posed by the aforementioned systems. As a result, domRBAC facilitates collaborations among domains under secure inter-operation, and provides support for resource usage management in the context of multi-domain computing environments, where mobile nodes operate as first-class entities.
\end{abstract}

Keywords: mobile Grid, role based access control (RBAC), secure interoperation, resource usage management, cross-domain authorization.

\section{Introduction}

In recent years, Grid computing has become the focal point of science and enterprise computer environments. The Grid is an emergent technology that can be defined as a system able to share resources and provide problem solving in a coordinated manner within dynamic, multi-institutional virtual organizations 9]. This definition depends mostly on the sharing of resources and the collaboration of individual users or groups within the same or among different virtual organizations, in a service oriented approach. In turn, mobile Grid systems incorporate additional complexity and new challenges, due to the support of dynamic virtual organizations and the commercialization of Grid services [25]. Access control, in 
such computing systems, is an active research area given the challenges and complex applications. The role of access control is to control and limit the actions or operations in a system performed by a user on a set of resources. In brief, it enforces the access control policy of a system and it prevents the access policy from subversion [3. An extensive research has been done in the area of access control in collaborative systems 24, 28. Nonetheless, further examination is demanded. This is mainly due to the partial or weak fulfilment of access control requirements in the aforementioned systems [11.

In this paper, we propose a new access control model called domRBAC to provide secure inter-operation among domains and resource usage management in collaborative systems, as the mobile Grid computing paradigm, where mobile devices can participate as first-class entities. Specifically we examine an incremental integration of individual RBAC policies into a global policy, which is suitable for dynamic virtual organizations. This is achieved via the definition of cross-domain mappings between roles. Thus, any user authorized for a role $d_{i} r_{m}$ in a domain $d_{i}$ is granted access to all the permissions of its mapped role $d_{j} r_{n}$ in domain $d_{j}$. Nevertheless, inter-domain collaborations is a challenging task since they can lead to various types of conflict. Research in [10] has shown that secure inter-operation in federated systems must conform to the principles of autonomy and security. The principle of autonomy states that any access permitted within an individual system must also be permitted under secure inter-operation. Regarding the principle of security, it states that any access not permitted within an individual system must also be denied under secure inter-operation [10]. The former principles can be preserved in a collaboration, if a number of violations are successfully identified. Violations in role-based approaches, possibly leading to privilege escalation, can occur due to conflicts in cyclic inheritance and in static and dynamic separation of duty relations. In regard to resource usage management, domRBAC provides the capability of applying usage policies and, thus, enforcing quality of service rules on sharable resources. The application of resource usage policies can greatly amplify the adoption of Grid systems. For instance, it can be applied in Grid systems where ad-hoc mobile devices operate as first-class entities, or in multi-tenant environments, where usage based pricing is required.

The structure of the remainder of this paper is as follows. Section 2 provides information on related work and presents our motivation. Section 3 discuss domRBAC model in a systematic manner. A demonstration of the proposed model is given in section 4. Finally, we present our concluding remarks in section 5 .

\section{Relevant Work and Motivation}

The access control models implemented by the existing Grid authorization mechanisms are either role based or attribute based. Role based access control (RBAC) approaches have gained considerable attention among researchers, due to ease of administration and support of a significant number of principles, namely the least privilege, separation of administrative functions and separation of duty relationships [20]. However, RBAC handles better centralized architectures and is 
rather weak in inter-domain collaborations. Such functionality is absent from the ANSI INCITS 359-2004 [2]. Attribute based access control (ABAC) approaches have lately gained a lot of attention due to the development of internet based distributed systems. ABAC can provide access decisions on resources based on the requestor's owned attributes. A basic advantage of ABAC in comparison to $\mathrm{RBAC}$ is that in the former approach it is possible to provide access to users in a collaborative environment without the need for them to be known by the resource a priori. The $\mathrm{UCON}_{A B C}$ model [15], [19] is a representative ABAC model, based on a modern conceptual framework, which encompasses traditional access control, trust management and digital rights management for the protection of digital resources.

Through time, numerous RBAC-based and ABAC-based models have been proposed trying to overcome some of the limitation of their initial implementations. In regard to RBAC and secure inter-operation, research in [21] proposed an integer programming (IP)-based approach for optimal resolution of the examined conflicts. A policy integration framework is used for the merging of the individual RBAC policies into a global policy. However, this approach is not dynamic, since the global policy is not a result of an incremental composition of the inter-domain policies. In [6] an inter-domain role-mapping approach based on the least privilege principle is suggested. Yet, the applied greedy algorithm may not compute optimal solutions, and from a security perspective may fail to find a safe solution. Research in 22 presents a protocol for secure inter-operation, which is based on the idea of access paths and access paths constraints. Nonetheless, the protocol does not check for violations during an inter-domain role assignment. Rather, it assumes that inter-domain role mappings already exist. In [26] the DRBAC is presented as a dynamic context-aware access control model for Grid applications. However, the management of inter-domain policies is not tackled. Resource usage management, to the best of our knowledge, is completely absent from the existing RBAC-based models. On the contrary, usage control was a subject of research in the UCON conceptual framework [15], [19, that is an ABAC model with the capability of enforcing RBAC policies. Nevertheless, UCON lacks administrative models and requires synchronized attribute acquisition and management that makes it more complex when applied to large systems.

In Grid systems, the existence of various access control models, inevitably led to the implementation of different Grid authorization mechanisms. Additionally, each mechanism tried to further implement features not intrinsically supported by the implemented model (i.e. support of inter-domain collaborations, quality of service and so on). Representative authorization mechanisms in Grid systems are the Community Authorization Service (CAS) [16], the Virtual Organization Membership Service (VOMS) 1, Akenti 23, PERMIS 4], 5], and Usage Based Authorization [27]. Regarding mobile Grid systems various architectures have been proposed to provide solutions, as the virtual cluster approach in [17], the mobile OGSI.NET [7] and the Akogrimo project [18. Yet, the proposed authorization mechanisms are complementary to existing Grid authorization services, as the $\mathrm{A} 4 \mathrm{C}$ infrastructure in Akogrimo. 
So far we have outlined the key requirements of mobile Grid systems for access control operation and administration, which are explicitly identified by the need for support of dynamic and secure inter-operation and interaction among the participating entities. To this extent, the examined access control models do not provide a solid solution to cope with these requirements of mobile Grid systems. Nonetheless, they are mostly targeted to general-purpose collaborative systems. Furthermore, the support of resource usage policies, in order to tackle the enforcement of quality of service policies is absent from RBAC family of models and only supported by the UCON conceptual framework. However, as discussed, ABAC solutions are prone to complexity when applied to large systems and lack administrative models. Therefore, we propose the domRBAC model, which combines the virtues of RBAC-based models and provides in addition resource usage management functionality in order to support modern Grid systems, as described in detail in the next section.

\section{The Proposed domRBAC Model for Modern Collaborative Systems}

The domRBAC model is an access control model capable of enforcing restrictive access control policies in collaborative systems, as the mobile Grid computing paradigm. The domRBAC model is based on the ANSI INCITS 359-2004 [2]. Thus, it supports all the components of the RBAC model, namely the core RBAC, hierarchical RBAC, static separation of duty relations, and dynamic of duty relations. However, domRBAC is enriched with additional functionality to cope with the requirements posed by modern computing environments. In this section, we discuss domRBAC model in a systematic manner.

\section{1 domRBAC Elements}

The domRBAC model consists of the following six basic elements: users, roles, sessions, operations, objects, and containers. Furthermore, domRBAC can support access control among domains. A domain can be defined as a protected computer environment, consisted of users and resources under an access control policy. This is done to cope with the problem of governing inter-operations among domains. Figure 1 illustrates the proposed access control model.

Sessions, objects and operations are three concepts that are commonly used in access control. The latter two form a new element of permissions. A permission or a privilege is an approval to perform an operation on one or more RBAC protected objects. In domRBAC, the aforementioned elements provide the same functionality in their familiar sense. As in all role-based models, sessions are dynamic elements. They are used as intermediary entities between the users and roles elements. The user element usually depicts a physical person who interfaces with a computer system. User elements, in role-based models, are assigned to role elements and vice-versa. Sessions, in role-based models, are used to enforce dynamic security policies to computing systems. Each user can be 


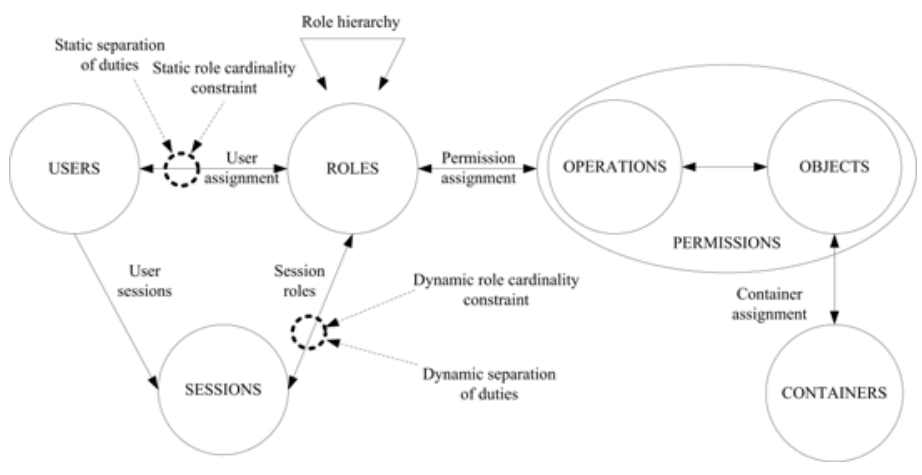

Fig. 1. The domRBAC access control model

associated with many sessions, and each session may have a combination of many active roles. Regarding objects, they are used to represent an entity in a computing system. Control of access to objects can be coarse-grained or finegrained, depending on the computing system. For instance, the sharing of files and exhaustible system resources can be considered as an example of coursegrained access control. On the contrary, the granting of access in a database on the level of record or field is an example of fine-grained access control. Yet, in domRBAC, an object can be associated with many container elements. The container element is explained in detail later in this section. Lastly, the element of operations provides a set of allowed operations on objects. Both operations and objects are system dependent. This means that different type of operations applies to different objects.

Roles in domRBAC are enriched with the notion of domains, and are expressed in pairs of domains and roles. For the naming of the roles, we use the DomainRole notation. Thus, the Domain prefix indicates the role's domain name, and the Role suffix indicates the name of the role. A formal definition is given later in definition 1.ii. The naming notation is used only for the element of roles. Nonetheless, when assigning users or permissions to roles, it is understood that the former two are also bound by the role's domain name. Through the role's naming convention, the domRBAC model can distinguish the security policies enforced among the autonomous domains.

The container is an abstract element that incorporates additional decision factors employed by the access decision function. The container can handle both environment and usage level information. The environment attributes are used to set time constraints, spatial information and so on and so forth. Yet, the usage level attributes can limit the usage of shared resources. The information specified in the container element is based on [14. Thus, a container attribute can represent a certain property of the environment or usage levels. A container function provides a mechanism to obtain the current value of a specific container attribute. Lastly, a container condition is a predicate that compares the current 
value of a container attribute either with a predefined constant, or another container attribute of the same domain. A significant enhancement of domRBAC as compared to the ANSI INCITS 359-2004 is that the element of container can support resource usage policies.

Moreover, domRBAC can support additional constraints, namely static and dynamic role cardinality constraints, which can be applied to the process of role assignment and role activation, respectively. This means that the number of roles that can be assigned to and/or activated by the users of a system can be managed. The constraint of role cardinality is introduced to fulfil both requirements posed by the system administrators as well as resource owners. Administrators can use static role cardinality to limit the assignment of critical roles with users. Furthermore, dynamic role cardinality can be used for setting quality of service rules. Resource owners can manage the usage of their resources by limiting the number of users that utilizes them. Thus, it is feasible to create license agreements between users and resource owners. This leads users to receive high quality services in a computing system.

Furthermore, the domRBAC model supports the identification of inter-domain violations, in an automated way, to avoid privilege escalation. The inter-domain violations are caused due to new immediate inter-domain role inheritance relations. The supported violations are: cyclic inheritance, violation of static separation of duty relations in a domain, and violation of dynamic separation of duty relations in a domain. Formal definitions are given later in this section in the Z formal description language [12] as in the ANSI INCITS 359-2004.

\section{2 domRBAC Definitions}

Definition 1. The core domRBAC.

The formal definition of core domRBAC model is based on [2], and is extended as follows:

i. USERS, ROLES, OPS, OBS, CNTRS, stands for users, roles, operations, objects and containers, respectively.

ii. $d_{\text {domain }} r_{\text {role }} \in$ ROLES is a role expressed in a DomainRole format, where Domain denotes a domain name and Role denotes a role name. For example, if a role $r_{m}$ belongs to a domain $d_{i}$, we write $d_{i} r_{m}$.

iii. $\mathrm{UA} \subseteq \mathrm{USERS} \times \mathrm{ROLES}$, a many-to-many mapping user-to-role assignment relation.

iv. assigned_users $\left(d_{i} r_{m}:\right.$ ROLES $) \rightarrow 2^{U S E R S}$, the mapping of role $d_{i} r_{m}$ onto a set of users.

Formal definition: assigned_users $\left(d_{i} r_{m}\right)=\left\{\mathrm{u} \in \operatorname{USERS} \mid\left(\mathrm{u}, d_{i} r_{m}\right) \in \mathrm{UA}\right\}$.

v. $\mathrm{PRMS}=2^{(O P S \times O B S)}$, the set of permissions.

vi. $\mathrm{PA} \subseteq \mathrm{PRMS} \times \mathrm{ROLES}$, a many-to-many mapping permission-to-role assignment relation.

vii. assigned_permissions $\left(d_{i} r_{m}:\right.$ ROLES $) \rightarrow 2^{P R M S}$, the mapping of role $d_{i} r_{m}$ onto a set of permissions.

Formal definition: assigned_permissions $\left(d_{i} r_{m}\right)=\left\{\mathrm{p} \in \operatorname{PRMS} \mid\left(\mathrm{p}, d_{i} r_{m}\right)\right.$ $\in \mathrm{PA}\}$. 
viii. $\mathrm{CA} \subseteq \mathrm{CNTRS} \times \mathrm{OBS}$, a many-to-many mapping container-to-object assignment relation.

ix. assigned_containers(o: OBS) $\rightarrow 2^{C N T R S}$, the mapping of object o onto a set of containers.

Formal definition: assigned_containers $(\mathrm{o})=\{\mathrm{c} \in \mathrm{CNTRS} \mid(\mathrm{c}, \mathrm{o}) \in \mathrm{CA}\}$.

$\mathrm{x} . \mathrm{Op}(\mathrm{p}: \mathrm{PRMS}) \rightarrow\{o p \subseteq O P S\}$, the permission to operation mapping, which gives the set of operations associated with permission $\mathrm{p}$.

xi. $\mathrm{Ob}(\mathrm{p}: \mathrm{PRMS}) \rightarrow\{o b \subseteq O B S\}$, the permission to object mapping, which gives the set of objects associated with permission $\mathrm{p}$.

xii. $\mathrm{SESSIONS}=$ the set of sessions.

xiii. session_user(s: SESSIONS) $\rightarrow$ USERS, the mapping of session s onto a corresponding user.

xiv. session_roles(s: SESSIONS) $\rightarrow 2^{R O L E S}$, the mapping of session s onto a set of roles.

Formal definition: session_roles $(s) \subseteq\left\{d_{i} r_{m} \in \operatorname{ROLES} \mid\right.$ (session_user $\left.(s), d_{i} r_{m}\right)$ $\in \mathrm{UA}\}$.

xv. avail_session_perms(s: SESSIONS) $\rightarrow 2^{P R M S}$, the permissions available to a user in a session $=\bigcup_{d_{i} r_{m} \in \text { session_roles(s) }}$ assigned_permissions $\left(d_{i} r_{m}\right)$.

Definition 2. Hierarchical domRBAC.

The hierarchical domRBAC is defined to cope with inter-domain role inheritance relations, and is enriched with notations from the theory of graphs. The reason why we choose to use the latter type of notation is bilateral. Firstly, graphs help in the visualisation of inter-domain role inheritance relations. Secondly, adjacency matrixes make it easy to find sub-graphs and adjacency queries are fast. Henceforth, we use $i$ and $j$ to refer to domains, where $i=j$ if we refer to an intra-domain relation, and $i \stackrel{ \pm}{=} j$ if we refer to inter-domain relations $($ intra - domain $\subseteq$ inter - domain $)$.

i. $R H \subseteq R O L E S \times R O L E S$ is a partial order on ROLES called the inheritance relation, written as $\geq$, where $d_{i} r_{m} \geq d_{j} r_{n}$ only if all permissions of $d_{j} r_{n}$ are also permissions of $d_{i} r_{m}$, and all users of $d_{i} r_{m}$ are also users of $d_{j} r_{n}$, i.e., $d_{i} r_{m} \geq d_{j} r_{n}$.

$\Rightarrow$ authorized_permissions $\left(d_{j} r_{n}\right) \subseteq$ authorized_permissions $\left(d_{i} r_{m}\right)$.

ii. authorized_users $s_{(i, j)}\left(d_{i} r_{m}: R O L E S\right) \rightarrow 2^{U S E R S}$, the mapping of role $d_{i} r_{m}$ onto a set of users on the presence of a role hierarchy.

Formal definition: authorized_users $s_{(i, j)}\left(d_{i} r_{m}\right)=\left\{u \in U S E R S \mid d_{j} r_{n} \geq\right.$ $\left.d_{i} r_{m},\left(u, d_{j} r_{n}\right) \in U A\right\}$.

iii. authorized_permissions ${ }_{(i, j)}\left(d_{i} r_{m}: R O L E S\right) \rightarrow 2^{P R M S}$, the mapping of role $d_{i} r_{m}$ onto a set of permissions in the presence of a role hierarchy.

Formal definition: authorized_permissions $(i, j)\left(d_{i} r_{m}\right)=\left\{p \in P R M S \mid d_{j} r_{n} \geq\right.$ $\left.d_{i} r_{m},\left(p, d_{j} r_{n}\right) \in P A\right\}$.

iv. $G=(V, E)$ is the inter-domain role hierarchy directed graph, which consists of a finite, nonempty set of role vertices $V \subseteq R O L E S$ and a set of edges $E$. Each edge is an ordered pair $\left(d_{i} r_{m}, d_{j} r_{n}\right), i \stackrel{ \pm}{=} j$ of role vertices that indicates the following relation: $d_{i} r_{m} \geq d_{j} r_{n}$. 
v. A path in a $G$ graph is a sequence of edges $\left(d_{i} r_{1}, d_{i} r_{2}\right),\left(d_{i} r_{2}, d_{i} r_{3}\right), \ldots$, $\left(d_{i} r_{n-1}, d_{i} r_{n}\right)$. This path is from role vertex $d_{i} r_{1}$ to role vertex $d_{i} r_{n}$ and has length $\mathrm{n}-1$. The path represents not immediate inheritance relation between role vertex $d_{i} r_{1}$ and $d_{i} r_{n}$.

vi. $A_{G}$ is the adjacency matrix representation for graph $G=(V, E)$, which is a $|V| \times|V|$ matrix, where $A_{G}\left[d_{i} r_{m}, d_{j} r_{n}\right]=1$ if there is an edge from role vertex $d_{i} r_{m}$ to role vertex $d_{j} r_{n}$, and $A_{G}\left[d_{i} r_{m}, d_{j} r_{n}\right]=0$ otherwise.

vii. Given a directed graph $G=(V, E)$ with adjacency matrix $A_{G}$, we compute a boolean matrix $T_{G}$ such that $T_{G}\left[d_{i} r_{m}, d_{j} r_{n}\right]$ is 1 if there is a path from $d_{i} r_{m}$ to $d_{j} r_{n}$ of length 1 or more, and 0 otherwise. We call $T$ the transitive closure of the adjacency matrix. For the computation of the transitive closure, the algorithm in 13 can be used. The algorithm computes the transitive closure of $G$ in $O\left(|V|^{3}\right)$ time and $O\left(|V|^{2}\right)$ space.

viii. An adjacency list representation for graph $G=(V, E)$ is an array $L$ of $|V|$ lists, one for each role vertex in $V$. For each role vertex $d_{i} r_{m}$, there is a pointer $L_{d_{i} r_{m}}$ to a linked list containing all the role vertices that are adjacent to $d_{i} r_{m}$.

ix. DESCENDANT_ROLES $S_{d_{i} r_{m}} \subseteq R O L E S$ is a set that contains the role vertices of adjacency list $L_{d_{i} r_{m}}$. Thus, DESCENDANT_ROLES $S_{d_{i} r_{m}}$ contains all the roles in the inter-domain collaboration that are immediate or not immediate descendant roles of a given role $d_{i} r_{m}$.

Definition 3. Constrained domRBAC.

Apart from the support of static and dynamic separation of duty constraints in each domain, domRBAC supports static and dynamic role cardinality constraints. Static role cardinality constraints can restrict the number of users assigned to a role, to a maximum number. Moreover, dynamic role cardinality constraints can restrict the number of users that activate a role, to a maximum number in all concurrent sessions. In the following, we redefine SSD and DSD in the presence of domains, and we define static and dynamic role cardinality.

i. Static Separation of duty (SSD): $\mathrm{SSD} \subseteq\left(2^{R O L E S} \times N\right)$ is a collection of pairs $\left(d_{i} r s, \mathrm{n}\right)$ in $\mathrm{SSD}$, where each $d_{i} r s$ is a role set in a domain $d_{i}, t$ a subset of roles in $d_{i} r s$, and $n$ is a natural number $\geq 2$, with the property that no user of domain $d_{i}$ is assigned to $\mathrm{n}$ or more roles from the set $d_{i} r s$ in each $\left(d_{i} r s, \mathrm{n}\right) \in \mathrm{SSD}$.

Formal definition:

$\forall\left(d_{i} r s, n\right) \in S S D, \forall t \subseteq d_{i} r s:|t| \geq n \Rightarrow \bigcap_{d_{i} r_{m} \in t}$ assigned_users $\left(d_{i} r_{m}\right)=\emptyset$.

ii. SSD in the presence of a hierarchy: In the presence of a role hierarchy SSD is redefined based on authorized users rather than assigned users as follows:

Formal definition:

$\forall\left(d_{i} r s, n\right) \in S S D, i=j, \forall t \subseteq d_{i} r s:|t| \geq n$

$\Rightarrow \bigcap_{d_{i} r_{m} \in t}$ authorized_users ${ }_{(i, j)}\left(d_{i} r_{m}\right)=\emptyset$. 
iii. Dynamic Separation of Duty (DSD): DSD $\subseteq\left(2^{R O L E S} \times N\right)$ is a collection of pairs $\left(d_{i} r s, \mathrm{n}\right)$ in DSD, where each $d_{i} r s$ is a role set and $\mathrm{n}$ a natural number $\geq 2$, with the property that no subject may activate $\mathrm{n}$ or more roles from the set $d_{i} r s$ in each $d s d \in$ DSD.

Formal definition:

$\forall d_{i} r s \in 2^{R O L E S}, n \in \mathbb{N},\left(d_{i} r s, n\right) \in D S D \Rightarrow n \geq 2 .\left|d_{i} r s\right| \geq n$, and $\forall s \in S E S S I O N S, \forall d_{i} r s \in 2^{R O L E S}$, $\forall$ role_subset $\in 2^{R O L E S}, \forall n \in \mathbb{N},\left(d_{i} r s, n\right) \in D S D$, role_subset $\subseteq d_{i} r s$, role_subset $\subseteq$ session_roles $(s) \Rightarrow \mid$ role_subset $\mid<n$.

iv. Static role cardinality (SRC): If static role cardinality constraint is required for any role $d_{i} r_{m}$, then $d_{i} r_{m}$ cannot be assigned to more than a maximum number of users.

$\mathrm{SRC} \subseteq(R O L E S \times N)$ is a collection of pairs $\left(d_{i} r_{m}, \mathrm{n}\right)$ in static role cardinality, where $d_{i} r_{m}$ is a role $r_{m}$ in a domain $d_{i}$ and $\mathrm{n}$ is a natural number $\geq 0$, with the property that the number of users assigned with role $d_{i} r_{m}$ cannot exceed the number $\mathrm{n}$ in each $\left(d_{i} r_{m}, \mathrm{n}\right) \in \mathrm{SRC}$.

Formal definition:

$d_{i} r_{m} \in R O L E S, n \in \mathbb{N}, n \geq 0$, $\forall\left(d_{i} r_{m}, n\right) \in S R C \Rightarrow \mid$ assigned_users $\left(d_{i} r_{m}\right) \mid \leq n$.

$\mathrm{v}$. SRC in the presence of a hierarchy: In the presence of a role hierarchy static role cardinality constraint is redefined based on authorized users rather than assigned users as follows:

$d_{i} r_{m} \in R O L E S, i \stackrel{+}{=} j, n \in \mathbb{N}, n \geq 0$, $\forall\left(d_{i} r_{m}, n\right) \in S R C \Rightarrow \mid$ authorized_user $s_{(i, j)}\left(d_{i} r_{m}\right) \mid \leq n$.

vi. Dynamic role cardinality constraint (DRC): If dynamic role cardinality is required for any role $d_{i} r_{m}$, then $d_{i} r_{m}$ cannot be activated for more than a maximum number of authorized users in all concurrent sessions of a system.

$\mathrm{DRC} \subseteq(R O L E S \times N)$ is a collection of pairs $\left(d_{i} r_{m}, \mathrm{n}\right)$ in dynamic role cardinality, where $d_{i} r_{m}$ is a role $r_{m}$ and $\mathrm{n}$ is a natural number $\geq 0$, with the property that the number of concurrent role activations by users authorized for role $d_{i} r_{m}$ cannot exceed the number $\mathrm{n}$.

Formal definition:

$d_{i} r_{m} \in R O L E S, n \in \mathbb{N}, n \geq 0$,

$\forall s \in \mathrm{SESSIONS},\left(d_{i} r_{m}, \mathrm{n}\right) \in \mathrm{DRC} \Rightarrow \sum \mid d_{i} r_{m} \cap$ session_roles $(s) \mid \leq n$.

After defining both the container element and the DRC constraint, we elaborate on the supported types of resource usage policies. The first type is via the container element, by declaring the required attribute value, function and condition of the container. However, this type of resource usage policy is unable to provide quality of service to consumers, since each container element restricts the usage of a resource on per role activation. A second type of resource usage policy with quality of service capabilities is provided via the combination of the container element and DRC constraint. This type of resource usage policy enforcement restricts the usage of a resource on all concurrent role activations. 
Definition 4. Role Inheritance Management in domRBAC.

The domRBAC model aims at providing a comprehensive solution to secure inter-operation based on the principles of autonomy, security and containment. In order to establish a secure inter-operation among the participating domains, domRBAC provides two new administrative commands for managing interdomain role inheritance relations. The administrative commands can be used by the administrator of each domain, according to the inter-operability requirements of each system. Their objective is to check for a number of violations before committing an inter-domain role inheritance relation. Thus, based on the definitions 4.i, 4.ii and 4.iii, we introduce the InterdomainPolicyViolation function for the checking of inter-domain violations due to the inter-domain role inheritance relations, and two new inter-domain administrative commands AddInterdomainInheritance and DeleteInterdomainInheritance for establishing and discarding immediate inter-domain inheritance relationships, respectively. Our approach utilizes algorithms derived from the theory of graphs. Intra-domain management not listed below is handled the same as in the ANSI INCITS 359-2004.

i. Inter-domain violation of role assignment: As stated in 21] an interdomain policy causes a violation of role assignment constraint of domain $d_{i}$ if it is allowed to a user $u$ of domain $d_{i}$ to access a local role $d_{i} r_{m}$ even though $u$ is not directly assigned to $d_{i} r_{m}$ or any of the roles that are senior to $d_{i} r_{m}$ in the role hierarchy of domain $d_{i}$.

We identify role assignment violations by checking for cyclic inheritance in the inter-domain role hierarchy graph. Role assignment violations can occur due to the addition of a new immediate inter-domain inheritance relationship $d_{i} r_{m_{a s c}} \gg d_{j} r_{n_{\text {desc }}}$ between existing roles $d_{i} r_{m_{a s c}}, d_{j} r_{n_{\text {desc }}}$, where $d_{i} r_{m_{\text {asc }}}$ is a role ascendant of $d_{j} r_{n_{d e s c}}$.

The algorithm for detecting inter-domain violations of role assignment is given in Table 1 .

ii. Intra-domain violation of SSD relationships: An inter-domain policy causes an intra-domain violation of SSD relationships of domain $d_{i}$ if it is allowed to a user $u$ of domain $d_{i}$ to be assigned to any two conflicting roles

Table 1. Inter-domain violation of role assignment algorithm

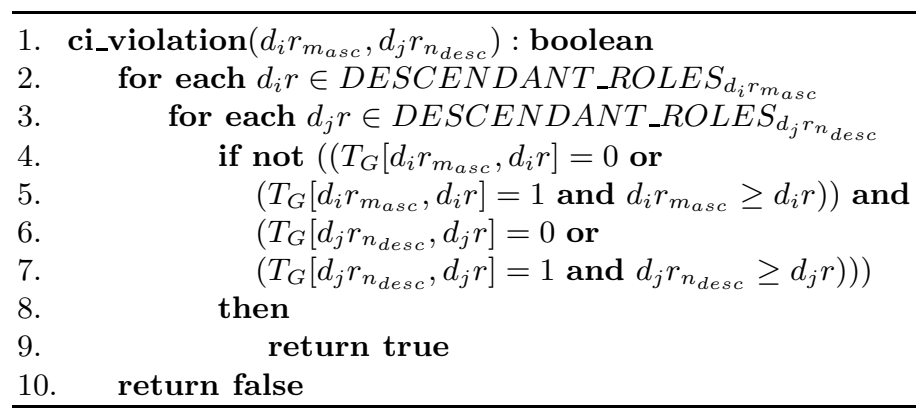


$d_{i} r_{m}$ and $d_{i} r_{n}$ of domain $d_{i}$. We identify violations of SSD relationships, using the following properties [8]:

Property 1: If there are two roles $d_{i} r_{m}$ and $d_{j} r_{n}$ that are mutually exclusive, then neither one should inherit the other, either directly or indirectly.

Property 2: If there are two roles $d_{i} r_{m}$ and $d_{j} r_{n}$ that are mutually exclusive, then there can be no third role that inherits both of them.

The algorithm for detecting intra-domain violations of SSD relationships is given in Table 2. Specifically, Property 1 is maintained in lines 6-7, and Property 2 in lines 8-9.

Table 2. Intra-domain violation of SSD relationships

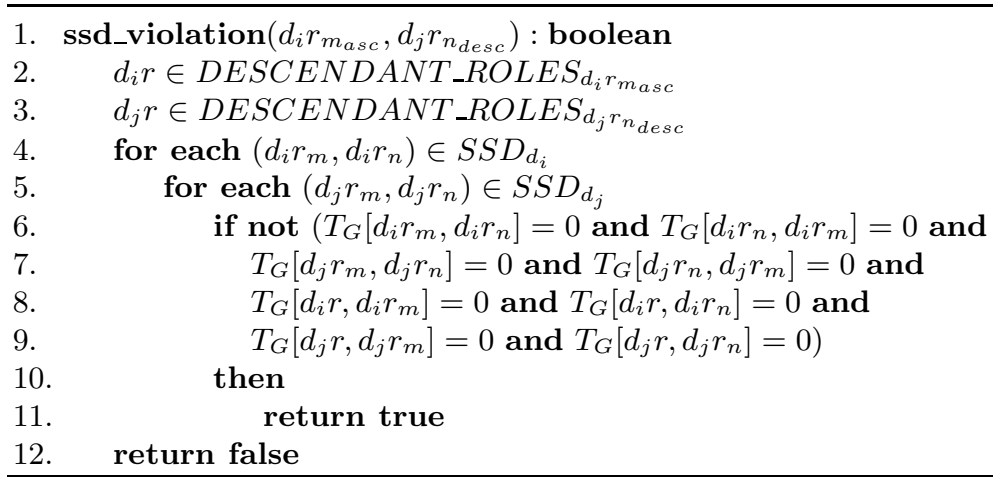

iii. Intra-domain violation of DSD relationships: An inter-domain policy causes an intra-domain violation of DSD relationships of domain $d_{i}$ if it is allowed to a user $u$ of domain $d_{i}$ to activate any two conflicting roles $d_{i} r_{m}$ and $d_{i} r_{n}$ of domain $d_{i}$. We identify violations of DSD relationships similarly to definition 4.ii due to the following property [8]:

Property 3: If SSD holds, then DSD is maintained. Thus, properties 1 and 2 must be guaranteed.

The algorithm for detecting intra-domain violations of DSD relationships is given in Table 3 .

iv. InterdomainPolicyViolation: This function checks if violations 4.i, 4.ii and 4.iii occur during an inter-domain role inheritance relation. It returns true if a violation occurs from an inter-domain role association, and false otherwise. Table 4 presents the implementation of the function.

v. AddInterdomainInheritance: This command establishes a new immediate inter-domain inheritance relationship $d_{i} r_{m_{a s c}} \gg d_{j} r_{n_{\text {desc }}}$ between existing roles $d_{i} r_{m_{a s c}}, d_{j} r_{n_{\text {desc }}}$. The command is valid if and only if $d_{i} r_{m_{a s c}}$ and $d_{j} r_{n_{\text {desc }}}$ are members of the ROLES dataset, $d_{i} r_{m_{a s c}}$ is not an immediate ascendant of $d_{j} r_{n_{d e s c}}$, and violations of role assignment and of SSD and DSD relationships do not occur. 
Table 3. Intra-domain violation of DSD relationships

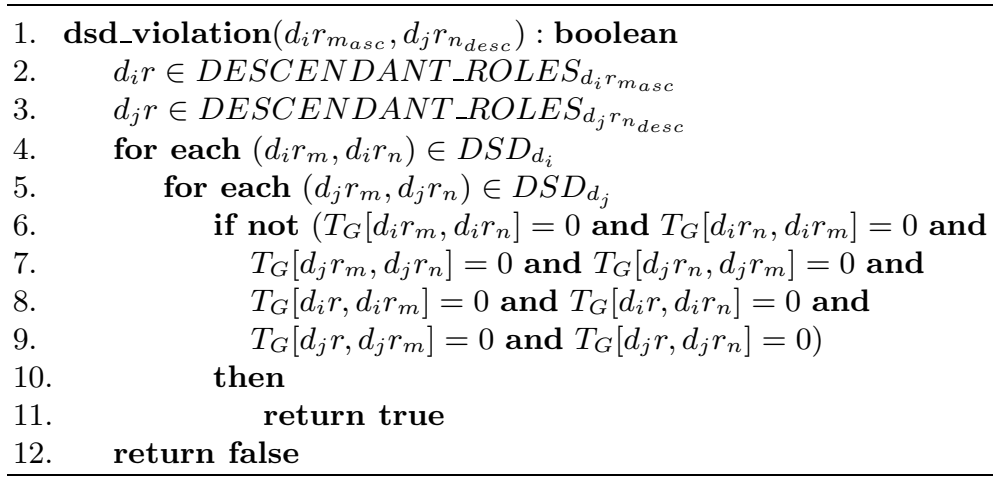

Table 4. Inter-domain policy violation function

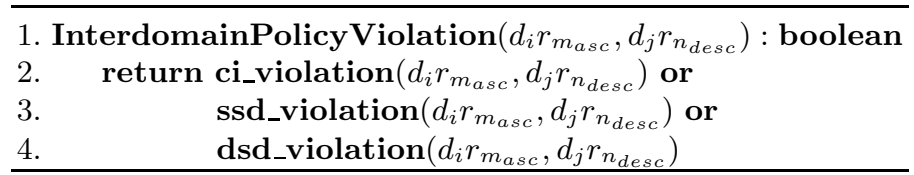

Formal definition:

AddInterdomainInheritance $\left(d_{i} r_{m_{a s c}}, d_{j} r_{n_{\text {desc }}}: N A M E\right) \triangleleft$

$d_{i} r_{m_{a s c}}, d_{j} r_{n_{\text {desc }}} \in R O L E S$;

InterdomainPolicyViolation $\left(d_{i} r_{m_{a s c}}, d_{j} r_{n_{\text {desc }}}\right)=$ false;

$\neg\left(d_{i} r_{m_{a s c}} \gg d_{j} r_{n_{\text {desc }}}\right) ; \neg\left(d_{j} r_{n_{\text {desc }}} \geq d_{i} r_{m_{a s c}}\right)$

$\geq^{\prime}=\geq \cup\left\{d r, d q: R O L E S \mid d r \geq d_{i} r_{m_{a s c}} \wedge d_{j} r_{n_{\text {desc }}} \geq d q \bullet d r \mapsto d q\right\} \triangleright$

vi. DeleteInterdomainInheritance: This command deletes an existing immediate inter-domain inheritance relationship $d_{i} r_{m_{a s c}} \gg d_{j} r_{n_{\text {desc }}}$. The command is valid if and only if the roles $d_{i} r_{m_{a s c}}$ and $d_{j} r_{n_{\text {desc }}}$ are members of the ROLES dataset, and $d_{i} r_{m_{a s c}}$ is an immediate ascendant of $d_{j} r_{n_{\text {desc }}}$. The new inter-domain inheritance relation is computed as the reflexive-transitive closure of the immediate inheritance relation resulted after deleting the relationship $d_{i} r_{m_{a s c}} \gg d_{j} r_{n_{\text {desc }}}$.

Formal definition:

DeleteInterdomainInheritance $\left(d_{i} r_{m_{a s c}}, d_{j} r_{n_{\text {desc }}}: N A M E\right) \triangleleft$

$d_{i} r_{m_{a s c}}, d_{j} r_{n_{\text {desc }}} \in \operatorname{ROLES} ;\left(d_{i} r_{m_{a s c}} \gg d_{j} r_{n_{\text {desc }}}\right)$

$\geq^{\prime}=\left(\gg\left\{d_{i} r_{m_{a s c}} \mapsto d_{j} r_{n_{\text {desc }}}\right\}\right)^{*} \triangleright$

\section{Use Cases}

In this section, we describe two contrived use cases to demonstrate the newly introduced functionality of domRBAC. The first use case demonstrates how to 
enforce resource usage policies, and the second how to identify security violations in an inter-domain role inheritance relation.

\subsection{Use Case 1: Resource Usage Management}

Figure 2(a) shows a simple policy in a domain $d_{1}$. Role $d_{1} r_{a}$ is a role senior to $d_{1} r_{b}$. User Alice requires to share the CPU cycles of her mobile device. Since the CPU capabilities of the device are limited, she decides to share only $50 \%$ of her CPU cycles, and to provide to each consumer at most $5 \%$ of her sharable CPU cycles. In order to apply the aforementioned policy, role $d_{1} r_{b}$ is assigned to permission $P_{U C}=(U$ sage, $C P U)$. This means that a usage operation is assigned to a CPU object. A container $c_{B}$ is assigned to object CPU. Container $c_{B}$ has the following properties: a container attribute that defines the CPU usage value equal to $5 \%$, a container function that returns the current CPU usage, and a container condition $\leq$. Moreover, a DRC constraint is applied to limit the number of active users to $10\left(D R C_{d_{1} r_{b}}=\left(d_{1} r_{b}, 10\right)\right)$. The latter constraint assures that the number of concurrent active users cannot exceed the 10 users. Thus, in conjunction with the container element it is assured that the usage of CPU not exceed the $50 \%$, and that each consumer receive at most $5 \%$ of CPU. If the DRC constraint was omitted, Alice would not be able to limit the usage of her resources, nor guarantee $5 \%$ of CPU usage to the consumers.

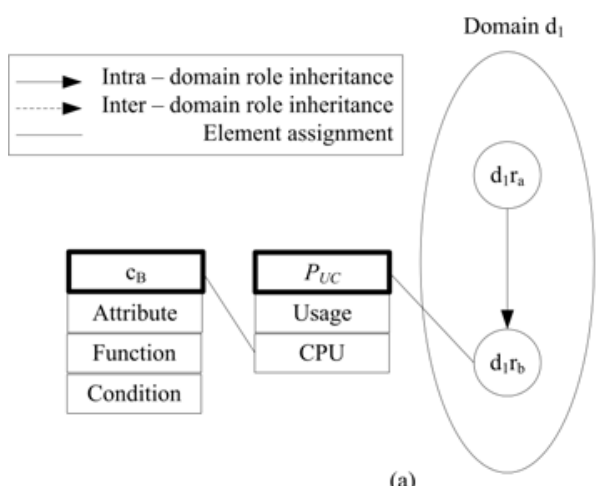

(a)

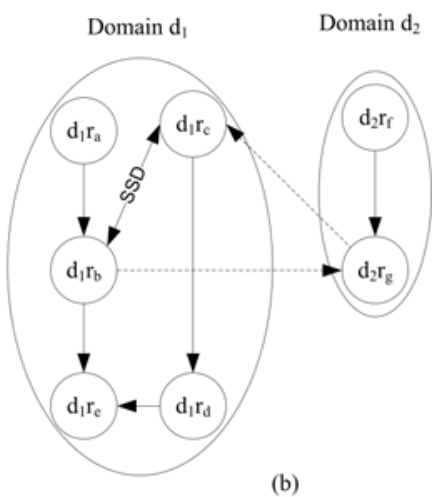

(b)

Fig. 2. (a) Resource usage management use case. (b) Security violation use case.

\subsection{Use Case 2: Security Violation}

Figure 2(b) shows a multi-domain policy that allows collaboration between domain $d_{1}$ and domain $d_{2}$. Domain $d_{1}$ has the following roles: $d_{1} r_{a}, d_{1} r_{b}, d_{1} r_{c}$, $d_{1} r_{d}$ and $d_{1} r_{e}$. Role $d_{1} r_{a}$ inherits all permissions of $d_{1} r_{b}$ which further inherits $d_{1} r_{e}$. Role $d_{1} r_{c}$ inherits all permissions of $d_{1} r_{d}$ which further inherits $d_{1} r_{e}$. A static separation of duty relation is specified for $d_{1} r_{b}$ and $d_{1} r_{c}$ meaning that these roles cannot be assigned to the same user simultaneously. Domain $d_{2}$ has 
the following roles: $d_{2} r_{f}$ and $d_{2} r_{g}$. Role $d_{2} r_{f}$ inherits all permissions of $d_{2} r_{g}$. The policy defines the following inter-operation between domains $d_{1}$ and $d_{2}$.

i. Role $d_{1} r_{b}$ inherits all the permissions available to role $d_{2} r_{g}$.

ii. Role $d_{2} r_{g}$ inherits all the permissions available to role $d_{1} r_{c}$.

However, the multi-domain policy leads to a violation of a SSD relationship in domain $d_{1}$. It allows $d_{1} r_{b}$ to access the permissions of role $d_{1} r_{c}$ through $d_{2} r_{g}$. Policy i does not raise any of the discussed violations. Regarding policy ii we work as follows:

Step 1. We assume that policy ii can be enforced.

Step 2. We construct the adjacency matrix $A_{G}$, which contains the inter-domain role hierarchy and we compute the sets of descendant roles for the two roles used in the multi-domain policy.

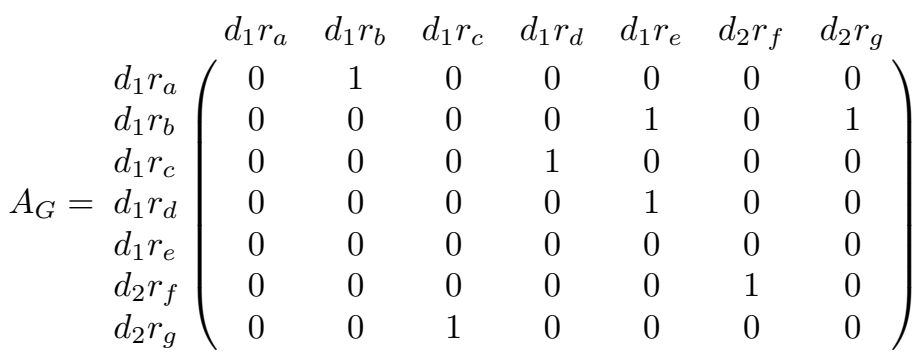

$D E S C E N D A N T \_R O L E S_{d_{2} r_{g}}=\left\{d_{1} r_{c}, d_{1} r_{d}, d_{1} r_{e}\right\}$, and DESCENDANT_ROLES $S_{d_{1} r_{c}}=\left\{d_{1} r_{d}, d_{1} r_{e}\right\}$.

Step 3. We call function InterdomainPolicyViolation with function parameters $d_{2} r_{g}$ and $d_{1} r_{c}$, and we compute the $s s d_{-} v i o l a t i o n\left(d_{2} r_{g}, d_{1} r_{c}\right)$. Based on definition 4.ii: $T_{G}\left[d_{1} r_{b}, d_{1} r_{c}\right]=1 \Rightarrow$ ssd_violation $\left(d_{2} r_{g}, d_{1} r_{c}\right)=$ true. The identification of a inter-domain violation of SSD relation will discard the inter-domain inheritance relationship, assumed in the hypothesis of step 1.

\section{Conclusion}

The proposed domRBAC model is an extended RBAC model with enhancements stemmed from a list of requirements from mobile Grid systems and commercialized applications. The applied enhancements result in a robust, scalable and dynamic access control model. The domRBAC model takes advantage of all the virtues of RBAC family of models, and additionally, encompasses features from ABAC approaches such as multi-domain support and resource usage management. Opposed to existing solutions, secure inter-operation among domains in domRBAC is achieved by checking gradually and dynamically for violations in inter-domain role inheritance relations, as required by mobile Grid systems. Furthermore, resource usage management is firstly introduced in an RBAC-based access control model, as a requirement for enforcing quality of service policies. Future work includes the design of an architecture that will implement the proposed access control model and a performance study. 


\section{References}

1. Alfieri, R., Cecchini, R.L., Ciaschini, V., dell'Agnello, L., Frohner, A., Gianoli, A., Lõrentey, K., Spataro, F.: VOMS, an authorization system for virtual organizations. In: Fernández Rivera, F., Bubak, M., Gómez Tato, A., Doallo, R. (eds.) Across Grids 2003. LNCS, vol. 2970, pp. 33-40. Springer, Heidelberg (2004)

2. American National Standard Institute, I.: Ansi incits 359-2004, role based access control (2004)

3. Benantar, M.: Access Control Systems: Security, Identity Management and Trust Models. Springer-Verlag New York, Inc., New York (2005)

4. Chadwick, D.: Authorisation in grid computing. Information Security Technical Report 10(1), 33-40 (2005)

5. Chadwick, D., Otenko, A., Ball, E.: Role-based access control with x. 509 attribute certificates. IEEE Internet Computing 7(2), 62-69 (2003)

6. Chen, L., Crampton, J.: Inter-domain role mapping and least privilege. In: SACMAT 2007: Proceedings of the 12th ACM Symposium on Access Control Models and Technologies, pp. 157-162. ACM, New York (2007)

7. Chu, D.C., Humphrey, M.: Mobile ogsi.net: Grid computing on mobile devices. In: IEEE/ACM International Workshop on Grid Computing, pp. 182-191 (2004)

8. Ferraiolo, D.F., Kuhn, D.R., Chandramouli, R.: Role-Based Access Control. Artech House, Inc., Norwood (2003)

9. Foster, I., Kesselman, C., Tuecke, S.: The anatomy of the grid: Enabling scalable virtual organizations. International Journal of High Performance Computing Applications 15(3), 200 (2001)

10. Gong, L., Qian, X.: Computational issues in secure interoperation. IEEE Trans. Softw. Eng. 22(1), 43-52 (1996)

11. Gouglidis, A., Mavridis, I.: On the definition of access control requirements for grid and cloud computing systems. In: Doulamis, A., Mambretti, J., Tomkos, I., Varvarigou, T. (eds.) GridNets 2009. Lecture Notes of the Institute for Computer Sciences, Social Informatics and Telecommunications Engineering, vol. 25, pp. 19-26. Springer, Heidelberg (2010)

12. ISO/IEC-13568: Information technology z - formal specification notation - syntax, type system and semantics, international Standard (2002)

13. Jonathan, L., Gross, J.Y. (eds.): Handbook of Graph Theory (Discrete Mathematics and Its Applications), 1st edn. CRC, Boca Raton (2003)

14. Neumann, G., Strembeck, M.: An approach to engineer and enforce context constraints in an rbac environment. In: SACMAT 2003: Proceedings of the Eighth ACM Symposium on Access Control Models and Technologies, pp. 65-79. ACM, New York (2003)

15. Park, J., Sandhu, R.: The ucon abc usage control model. ACM Trans. Inf. Syst. Secur. 7(1), 128-174 (2004)

16. Pearlman, L., Welch, V., Foster, I., Kesselman, C., Tuecke, S.: A community authorization service for group collaboration. In: Proceedings of the Third International Workshop on Policies for Distributed Systems and Networks., pp. 50-59. IEEE, Los Alamitos (2002)

17. Phan, T., Huang, L., Dulan, C.: Challenge: integrating mobile wireless devices into the computational grid. In: Proceedings of the 8th Annual International Conference on Mobile Computing and Networking, p. 278. ACM, New York (2002)

18. Racz, P., Burgos, J., Inacio, N., Morariu, C., Olmedo, V., Villagra, V., Aguiar, R., Stiller, B.: Mobility and qos support for a commercial mobile grid in akogrimo. In: 16th IST on Mobile and Wireless Communications Summit, pp. 1-5 (2007) 
19. Sandhu, R., Park, J.: Usage control: A vision for next generation access control. In: Gorodetsky, V., Popyack, L.J., Skormin, V.A. (eds.) MMM-ACNS 2003. LNCS, vol. 2776, pp. 17-31. Springer, Heidelberg (2003)

20. Sandhu, R.S., Coyne, E.J., Feinstein, H.L., Youman, C.E.: Role-based access control models. IEEE Computer 29(2), 38-47 (1996)

21. Shafiq, B., Joshi, J.B.D., Bertino, E., Ghafoor, A.: Secure interoperation in a multidomain environment employing rbac policies. IEEE Trans. on Knowl. and Data Eng. 17(11), 1557-1577 (2005)

22. Shehab, M., Bertino, E., Ghafoor, A.: Serat: Secure role mapping technique for decentralized secure interoperability. In: SACMAT 2005: Proceedings of the Tenth ACM Symposium on Access Control Models and Technologies, pp. 159-167. ACM, New York (2005)

23. Thompson, M., Essiari, A., Mudumbai, S.: Certificate-based authorization policy in a pki environment. ACM Transactions on Information and System Security (TISSEC) 6(4), 566-588 (2003)

24. Tolone, W., Ahn, G.J., Pai, T., Hong, S.P.: Access control in collaborative systems. ACM Comput. Surv. 37(1), 29-41 (2005)

25. Waldburger, M., Stiller, B.: Regulatory issues for mobile grid computing in the european union. In: 17th European Regional ITS Conference, Amsterdam, The Netherlands, pp. 1-9 (2006)

26. Zhang, G., Parashar, M.: Dynamic context-aware access control for grid applications. In: Proceedings of the Fourth International Workshop on Grid Computing 2003, pp. 101-108. IEEE, Los Alamitos (2004)

27. Zhang, X., Nakae, M., Covington, M., Sandhu, R.: A usage-based authorization framework for collaborative computing systems. In: Proceedings of the 11th ACM Symposium on Access Control Models and Technologies, pp. 180-189. ACM, New York (2006)

28. Zhang, X., Nakae, M., Covington, M.J., Sandhu, R.: Toward a usage-based security framework for collaborative computing systems. ACM Trans. Inf. Syst. Secur. 11(1), 1-36 (2008) 